\title{
SINGLE-PARTICLE BEAM DYNAMICS STUDIES FOR THE UNIVERSITY OF MARYLAND ELECTRON RING
}

\author{
L.G. Vorobiev, X. Wu and R.C. York \\ NSCL, Michigan State University, East Lansing, MI 48824
}

\begin{abstract}
A single particle analysis without space charge of the University of Maryland Electron Ring was performed. Higher order multipole terms of the dipole magnets and quadrupole lenses were included. The influence of the Earth's magnetic field was balanced by Helmholtz-like wires and the misalignments effects were corrected by short dipoles (correctors) together with adjustment of the lattice dipoles. Mispowering studies showed that the closed orbit distortions and lattice functions variations were within acceptable limits.
\end{abstract}

\section{INTRODUCTION}

The goal of the Electron-Ring project is the construction of a compact, low-energy electron ring operating with very high current electron beams. Experiments with this facility should help improve our understanding of space charge dominated beam [1], and thus, support practical designs for projects such as Heavy Ion Driven Inertial Fusion and other advanced accelerator applications.

A necessary (but by no means sufficient) condition for the E-Ring is reliable operation at zero current. The present work is concentrated on single particle analysis in the absence of space charge. The lattice working point, the influence of magnet multipoles on beam dynamics, compensation of the Earth's magnetic field, as well as correction of effects due to mechanical misalignment were evaluated. The effects of magnet mispowering and overall influence of imperfections were also considered. The computational tool used was the DIMAD code [2].

A general layout of the E-Ring is shown in Figure 1. It has a circumference of $11.52 \mathrm{~m}$ and an injected beam rigidity of 3.39 gauss. $\mathrm{m}$. There are 36 dipoles (each bending $10^{\circ}$ ) and 72 quadrupole lenses. The one cell structure is FODO with the E-Ring consisting of 36 cells (=18 segments).

\section{LATTICE WORKING POINT}

According to preliminary estimates of the E-Ring design parameters, the focusing structure should have a phase advance of about $76^{\circ}$ per cell for a zero current beam. The working point $\left(v_{x}=7.78\right.$ and $\left.v_{y}=7.70\right)$ was chosen to avoid all resonances up to 4 th order. Since the estimated space charge tune shift for full current will be about
$\Delta v \approx 6.0$ (see [3]), the working point will drift through many resonances and will be a topic of further studies.

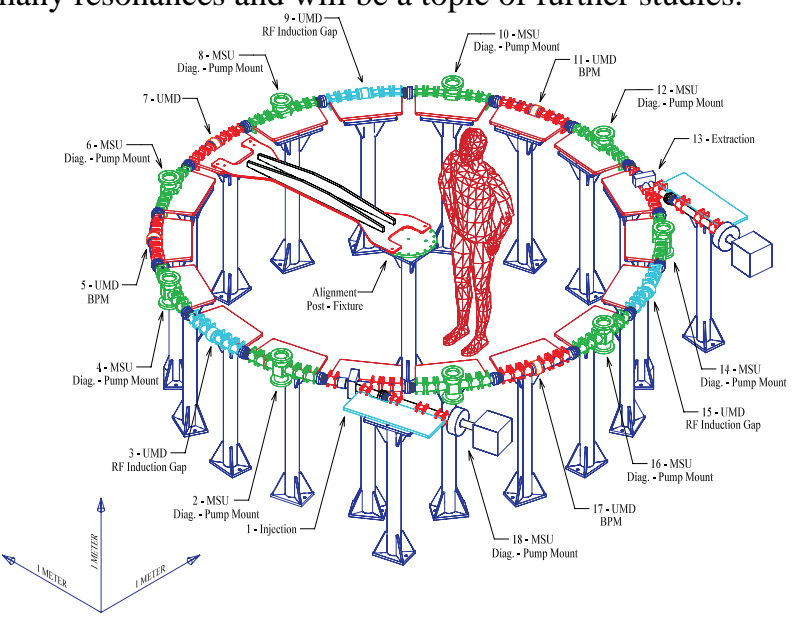

Figure 1. E-Ring layout.

Machine functions for a cell. Given the chosen $v_{x, y}$, machine functions of the E-Ring from DIMAD are shown in Figure 2 where the Twiss functions and dispersion are plotted for one segment (two cells). The matched machine functions are: $\beta_{\mathrm{x}}=0.261 \mathrm{~m}, \alpha_{\mathrm{x}}=-1.31, \beta_{\mathrm{y}}=0.264 \mathrm{~m}, \alpha_{\mathrm{y}}=$ $+1.32, \eta_{\mathrm{x}}=0.0358 \mathrm{~m}, \eta^{\prime}{ }_{\mathrm{x}}=0.1447$. The phase advances for one cell are $v_{x}=0.2161, v_{y}=0.2139$.

Smear. To check the choice of the working point, a quantitative criterion "the smear factor" was used to estimate the phase volume dilution after multi-turn tracking.

Single particle tracking (typically 1000 turns) produced a data array of phase coordinates $\left(x, x^{\prime}, y, y^{\prime}\right)$ after every turn. The Courant-Snyder invariants for each turn was then calculated from the machine functions and the phase coordinates at that point. The array of these invariants was then processed and the maximum $\left(A_{x, y}^{\max }\right)$, minimum $\left(A_{x, y}^{\text {min }}\right)$, and mean $\left(A_{x, y}^{\text {mean }}\right)$ invariant values were found [4]. The smear factors for the horizontal and vertical planes were calculated as: $S_{x, y}=\left(A_{x, y}^{\max }{ }_{x, y}^{\text {min }}{ }_{x, y}\right) / A_{x, y}^{\text {mean }}$.

For purely linear motion, the smear value is equal zero. In the presence of non-linear terms and/or mechanical misalignments, mispowering etc. the smear increases. From DIMAD tracking to $2^{\text {nd }}$ order, the total smear increase in both planes for the unperturbed lattice was found to be $<1.5 \%$ for an emittance of $10 \pi \mathrm{mm}$-mrad. 


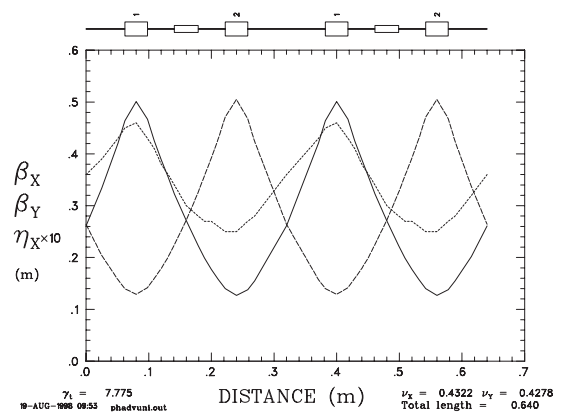

Figure 2. Machine functions ( $\beta \mathrm{x}-$ solid, $\beta \mathrm{y}$ - dashed, $\eta_{\mathrm{x}}$ - dots) for one segment ( 2 cells).

\section{MAGNET MULTIPOLES}

All the ring magnetic elements will be fabricated using "printed-circuit" technology. Magnetic measurement data for the dipoles and quadrupoles, obtained from the University of Maryland and included in the optics analysis. In the tracking, we assumed that all allowed multipoles were systematic (errors due to elements fabrication), whereas all errors corresponding to nonallowed multipoles (errors due to focusing structure assembling) were random.

Dipole multipole analysis. The magnetic field in the dipoles may be represented by $B^{D}=B \rho \sum_{n=0}^{n=4} K_{n}^{D} x^{n}$ where $B \rho=3.39$ gauss $\cdot \mathrm{m}$ is the beam magnetic rigidity and $K_{n}^{D}$ are the expansion coefficients. The value $K_{0}^{D}=B_{0}^{D} / B \rho=4.64[1 / \mathrm{m}]$ (with $\mathrm{B}_{0}{ }^{\mathrm{D}}=15.73$ gauss and an effective length of $37.6 \mathrm{~mm}$ providing a $10^{\circ}$ bend). For $\mathrm{n}>1$ one obtains $K_{n}^{D}=K_{0}^{D} B_{n}^{D} /\left(B_{0}^{D} R\right)$ and $\mathrm{B}_{\mathrm{n}}^{\mathrm{D}} / \mathrm{B}_{0}^{\mathrm{D}}$ are listed in Table 1.

Table 1. Dipole multipole measurements.

\begin{tabular}{||c|c|c|c||}
\hline \hline $\mathbf{N}$ & Amplitude $\boldsymbol{B}_{n}{ }^{\boldsymbol{D}} / \boldsymbol{B}_{\boldsymbol{0}}{ }^{\boldsymbol{D}}$ & $\boldsymbol{K}_{n}{ }^{\boldsymbol{1}}$ & Type \\
\hline 1 & $0.076 \%$ & $1.481 \mathrm{e}+00$ & Random \\
\hline 2 & $2.00 \%$ & $1.637 \mathrm{e}+02$ & Systematic \\
\hline 3 & $0.00 \%$ & 0 & Random \\
\hline 4 & $0.49 \%$ & $7.074 \mathrm{e}+04$ & Systematic \\
\hline \hline
\end{tabular}

Quadrupole multipole analysis. For the magnetic field of a quadrupole, we use a similar representation: $B^{Q}=B \rho \sum_{n=1}^{n=5} K_{n}^{Q} x^{n}$. The value $K_{l}^{Q}=227\left[1 / \mathrm{m}^{2}\right]$ was found from lattice fitting, and since $B_{l}^{Q}=B \rho K_{l}^{Q} R$ and $B_{n}^{Q}=$ $B \rho K_{n}^{Q} R^{n}$ and for $n>1$ are given by $K_{n}=K_{1}^{Q} B_{n}^{Q} /\left(R^{n-1} B_{1}\right)$. The quadrupole multipole values are listed in Table 2 .

Smear from Magnet Multipoles. The smear factor was used to estimate the influence of the higher-order multipoles on the beam dynamics of the E-Ring. All random errors were assumed to have a Gaussian distribution with $l \sigma$ corresponding to the value of the coefficients $K_{n}^{Q}$ for the random terms. The values for the systematic terms were fixed at the values given since their contribution to smear was found negligible even for several times the measured values. (See Tables 1 and 2).

Table 2. Quadrupole multipole analysis.

\begin{tabular}{||c|c|c|c||}
\hline \hline $\mathbf{N}$ & Amplitude $_{\mathbf{n}}{ }^{\mathbf{Q}} / \mathbf{B}_{1}{ }^{\mathbf{Q}}$ & $\mathbf{K}_{\mathbf{n}}{ }^{\mathbf{Q}}$ & Type \\
\hline 2 & $1.00 \%$ & $1.737 \mathrm{e}+00$ & Random \\
\hline 3 & $0.30 \%$ & $2.188 \mathrm{e}+02$ & Random \\
\hline 4 & $0.56 \%$ & $1.715 \mathrm{e}+00$ & Random \\
\hline 5 & $0.112 \%$ & $7.940 \mathrm{e}+05$ & Systematic \\
\hline
\end{tabular}

The smear $S_{x, y}$ was evaluated as a function of the multipole strength by taking the $\sigma$ value for the Gaussian distribution to be $1,2,3$, or 4 times the values given in Table 1 and Table 2. Even for random errors three times that measured, the smear is still below 5\% after 1000-turn tracking for a beam of emittance $10 \pi \mathrm{mm}$-mrad [3]. Hence, the measured dipole and quadrupole multipoles are adequate for the ring operation.

We found that random errors (non-allowed poles) are the major contributors to smear growth with the systematic errors providing minor contributions to the smear. The largest contributions were due to $K_{1}^{B}$ (the quadrupole component in the dipoles) and to $K_{l}^{Q}$ (the sextupole component in the quadrupoles).

\section{EARTH'S MAGNETIC FIELD}

Consistent with measurements by the University of Maryland group of the Earth's magnetic field in the vicinity of the ring, for the tracking analysis, the Earth's magnetic field was represented as:

$$
\begin{aligned}
& B_{r}=0.225 \sin \theta \text { [gauss] (the radial field) and } \\
& B_{z}=-0.55 \text { [gauss] (the vertical field) }
\end{aligned}
$$

Where $\theta$ is the azimuthal position around the ring.

The effect of the Earth's magnetic field was represented by 13 kicks per segment or 234 kicks around the ring. The vertical field was balanced by adjustment of the 36 ring dipole magnets. The radial field was compensated by 18 segments of Helmholtz-like conductors with the current of 4.6 A. Under these conditions (the multipole values of Table 1 and Table 2 are included), the analysis of the closed orbit distortion after compensation resulted in:

$$
\begin{aligned}
& \mathbf{x}_{\mathrm{rms}}=0.508 \mathrm{~mm}, \mathrm{y}_{\mathrm{rms}}=0.167 \mathrm{~mm} \\
& \mathrm{x}_{\text {max }}=0.868 \mathrm{~mm}, \mathrm{y}_{\text {max }}=0.453 \mathrm{~mm}
\end{aligned}
$$

\section{MISALIGNMENTS AND CORRECTIONS}

Misalignments of the ring magnetic elements were evaluated. The estimated probable misalignment values 
are given in Table 3 where $\Delta x, \Delta y, \Delta z$ denote shifts in the horizontal, vertical and longitudinal directions, respectively, and $\Delta \mathrm{z}^{\prime}$ is the rotation around the $\mathrm{z}$-axis. The mechanical misalignments were then represented by a Gaussian distribution with the $\sigma$ values of Table 3 .

Table 3. Mechanical misalignment values.

\begin{tabular}{||c|c||}
\hline Misalignment & $1 \sigma-$ value \\
\hline$\Delta \mathrm{x}$ & $0.1 \mathrm{~mm}$ \\
\hline$\Delta \mathrm{y}$ & $0.1 \mathrm{~mm}$ \\
\hline$\Delta \mathrm{z}$ & $1.0 \mathrm{~mm}$ \\
\hline$\Delta \mathrm{z}^{\prime}$ & $4.0 \mathrm{mrad}$ \\
\hline
\end{tabular}

The mechanical misalignments result in closed orbit distortions (CODs). The correction of these CODs requires beam position monitors (BPMs) combined with active correctors in both planes. The simulated correction scheme used the readjustment of the 36 ring dipole magnets for horizontal compensation and 36 vertical correctors placed between the dipoles and quadrupoles for the vertical correction. (The vertical corrector magnetic field strengths required were $<1$ gauss at the injection energy assuming an effective length of $1.7 \mathrm{~cm}$ ).

Under these conditions (multipole values of Tables 1-2 included, but without the effects of the Earth's magnetic field), the CODs were evaluated.

Table 4. CODs as a function of BPM number for the misalignment values of Table 3. *No BPMs.

\begin{tabular}{||c|c|c|c|c||}
\hline \hline $\begin{array}{c}\text { BPM } \\
\#\end{array}$ & $\begin{array}{c}\text { Xmax } \\
(\mathrm{mm})\end{array}$ & $\begin{array}{c}\text { Ymax } \\
(\mathrm{mm})\end{array}$ & $\begin{array}{c}\text { Xrms } \\
(\mathrm{mm})\end{array}$ & $\begin{array}{c}\text { Yrms } \\
(\mathrm{mm})\end{array}$ \\
\hline $0^{*}$ & 1.81 & 1.86 & 0.61 & 0.77 \\
\hline 15 & 1.14 & 1.34 & 0.34 & 0.44 \\
\hline 18 & 1.14 & 1.07 & 0.30 & 0.38 \\
\hline
\end{tabular}

We have explored three cases: CODs before correction, CODs after correction with 18 (one per segment) and 15 (one missing per segment) BPMs. In Table 4 for 15 BPMs, the CODs were evaluated as a function of the misalignment values. The sigma of the Gaussian distribution was taken to be 1,2 , and 3 times the values of Table 3. The results are given in Table 5.

Table 5. CODs values after correction with 15 BPMs as a function of the scaled misalignment values of Table 3 .

\begin{tabular}{|c|c|c|c|c||}
\hline $\begin{array}{c}\sigma \\
\text { scale factors }\end{array}$ & $\begin{array}{c}\text { Xmax } \\
(\mathrm{mm})\end{array}$ & $\begin{array}{c}\text { Ymax } \\
(\mathrm{mm})\end{array}$ & $\begin{array}{c}\text { Xrms } \\
(\mathrm{mm})\end{array}$ & $\begin{array}{c}\text { Yrms } \\
(\mathrm{mm})\end{array}$ \\
\hline 1 & 1.14 & 1.34 & 0.34 & 0.44 \\
\hline 2 & 2.28 & 2.62 & 0.70 & 0.88 \\
\hline 3 & 3.43 & 3.84 & 1.08 & 1.32 \\
\hline
\end{tabular}

\section{MAGNET MISPOWERING}

The beam dynamics will also be affected by mispowering of the ring magnetic elements. Mispowering of the dipole magnets will result in COD, whereas quadrupole mispowering will primarily cause variation of lattice functions and tune. The analysis was performed to determine the appropriate power supply specifications with the multipoles of Tables 1-2 (no other perturbations were included). The mispowering perturbations were considered systematic and the effect of mispowering was simulated by DIMAD assuming a systematic error in the momentum spread. The CODs as a function of the dipole mispowering and the $\beta \mathrm{x}, \mathrm{y}$ functions variance as a function of quadrupole mispowering showed that dipole and quadrupole power supplies with ripple specifications of $10^{-4}$ and $10^{-3}$ respectively, ensures acceptable performance.

\section{DISCUSSION AND CONCLUSIONS}

Finally, under the conditions:

- Magnet Multipoles (values of Tables 1-2.)

- Earth's Magnetic Fields and Corrections

- Mechanical Misalignments (the values of Table 3) and corrections with 15 BPMs

- Magnet Mispowering (set at $10^{-4}$ and $10^{-3}$ for the dipoles and quadrupoles respectively)

The overall CODs with all errors included simultaneously were:

$$
\begin{aligned}
& \mathrm{x}_{\mathrm{rms}}=1.0 \mathrm{~mm}, \mathrm{y}_{\mathrm{rms}}=0.47 \mathrm{~mm} \\
& \mathrm{x}_{\max }=2.3 \mathrm{~mm}, \mathrm{y}_{\max }=1.30 \mathrm{~mm} .
\end{aligned}
$$

We conclude that the choice of the focusing structure will provide adequate ring performance in the absence of space charge. An unusual feature of the E-Ring is the significant influence of the Earth's magnetic field. The Helmholz-like wires were found to be an efficient mechanism for compensation of the radial component of this field. Though not investigated, it might be possible to utilize the corrector dipole magnets (used to correct for the mechanical misalignments) in lieu of the Helmholz coils.

\section{REFERENCES}

[1] M.Reiser et al. Fusion Eng. Des. 32-33, 293 (1996). See also: http://www.ipr.umd.edu/ebte/ring/

[2] Users Guide to the Program DIMAD, SLAC Report 285 UC-28 (1985).

[3] L.G.Vorobiev, X.Wu and R.C.York, MSUCL-1114 Report, MSU/NSCL, East Lansing, September (1998).

[4] Physica Reference Manual, TRIUMF, Canada (1994). 